\title{
Effect of orientation on broadband acoustic scattering of Antarctic krill Euphausia superba: Implications for inverting zooplankton spectral acoustic signatures for angle of orientation
}

\author{
L. V. Martin Traykovski ${ }^{a}$ \\ Massachusetts Institute of Technology/Woods Hole Oceanographic Institution Joint Program in \\ Oceanography and Applied Ocean Sciences and Engineering, Woods Hole Oceanographic Institution, \\ MS 32, Woods Hole, Massachusetts 02543 \\ R. L. O'Driscoll \\ Department of Marine Science, University of Otago, P.O. Box 56, Dunedin, New Zealand \\ D. E. McGehee ${ }^{\text {b) }}$ \\ Department of Applied Ocean Physics and Engineering, Woods Hole Oceanographic Institution, \\ Woods Hole, Massachusetts 02543
}

(Received 26 January 1998; accepted for publication 16 June 1998)

\begin{abstract}
Acoustic scattering experiments involving simultaneous acquisition of broadband echoes and video footage from several Antarctic krill were carried out to determine the effect of animal orientation on echo spectral structure. A novel video analysis technique, applied to extract krill angle of orientation corresponding to each insonification, revealed that echo spectra from krill near broadside incidence relative to the incident acoustic wave exhibited widely spaced, deep nulls, whereas off-broadside echo spectra had a more erratic structure, with several closely spaced nulls of variable depth. The pattern of changes in echo spectra with orientation for the experimentally measured acoustic returns was very similar to theoretically predicted patterns based on a distorted wave Born approximation (DWBA) model. Information contained in the broadband echo spectra of the krill was exploited to invert the acoustic returns for angle of orientation by applying a newly developed Covariance Mean Variance Classification (CMVC) approach, using generic and animal-specific theoretical and empirical model spaces. The animal-specific empirical model space was best able to invert for angle of orientation. The CMVC inversion technique can be implemented using a generic empirical model space to determine angle of orientation based on broadband echoes from individual zooplankton in the field. (C) 1998 Acoustical Society of America. [S0001-4966(98)05109-1]
\end{abstract}

PACS numbers: 43.30.Sf, 43.30.Pc [DLB]

\section{INTRODUCTION}

Antarctic krill provide a direct trophic link between the primary producers and the top predators (seabirds, whales, seals) of the Southern Ocean (Everson, 1977; El-Sayed, 1988; Nemoto et al., 1988; Permitin, 1970). In addition, a commercially important krill fishery became established about 20 years ago, peaking in the early 1980s with landings of over 500000 metric tons (Nicol and de la Mare, 1993). Because Euphausia superba plays such a central ecological role in the Antarctic marine food web, affecting the breeding success of the top predators that rely on it as a food source (Croxall et al., 1988), it has become increasingly important to assess and manage the impact of the fishery on krill stocks. Consequently, accurate knowledge of krill distribution, abundance (biomass), and production is necessary in

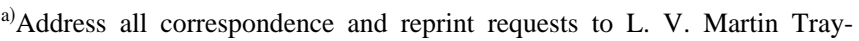
kovski, Woods Hole Oceanographic Institution, MS 32, Woods Hole, MA 02543, Electronic mail: lmartin@whoi.edu, Tel.: (508) 289-2750, Fax: (508) 457-2134.

${ }^{b}$ Present address: Tracor Applied Sciences, 4669 Murphy Canyon Road, Suite 102, San Diego, CA 92123.
}

order to characterize the trophic interactions in the Southern Ocean food web, as well as to successfully manage krill stocks as a resource.

Conventional methods for estimating zooplankton biomass include measurement of displacement volume, wet weight, dry weight, or carbon (see Wiebe et al., 1975) from net (e.g., MOCNESS-Wiebe et al., 1985) or pump (Miller and Judkins, 1981) samples. As a result of the spatial patchiness of zooplankton populations in the ocean and extreme temporal variability in their abundance, it is estimated that there can be as much as seven orders of magnitude of variability in biomass on the spatial and temporal scales important for populations of macrozooplankton (Huntley and Lopez, 1992). For example, Antarctic krill are distributed over a vast area of ocean, aggregating in patches, shoals, schools, swarms, and superswarms, which can cover many square km and extend to 200-m depth, displaying complex, small-scale, horizontal, and vertical structure (Nicol and de la Mare, 1993). Conventional techniques for biomass estimation (nets, pumps, trawls) are not suited for simultaneous sampling of the entire water column over the relevant scales, nor to resolving ecologically important small-scale patterns of krill distribution. To make more accurate biomass estimates, 
high-resolution $(\sim 1 \mathrm{~m})$ instruments capable of mapping variation in zooplankton biomass on large vertical (10-100 $\mathrm{m}$ ), horizontal (100-1000 km), and temporal (days to months) scales are required. The use of high-frequency acoustics to make volume backscatter measurements of the water column has made it possible to do rapid, highresolution, broad-scale synoptic surveys of krill abundance over the time and space scales of interest.

Traditional acoustic biomass estimation methods have employed single-frequency acoustic measures in conjunction with either theoretical models (e.g., Greenlaw, 1979) or empirical regression relationships between acoustic backscatter and biomass collected in simultaneous net samples (e.g., Flagg and Smith, 1989). Attempts to use volume backscatter measurements of the ocean as indicators of zooplankton type, size, and biomass rely on the accurate acoustic characterization of the zooplankton species of interest. Biomass estimates based on simple regression curves or on singlefrequency echo energy measurements may be subject to large errors, particularly if important factors such as speciesspecific material properties, morphology, and animal orientation are overlooked. Much effort has been put toward characterizing the acoustic target strength of krill for the purposes of species identification, animal size classification, abundance estimation, and acoustic signal separation. Singlefrequency target strength measurements have been made of krill and other elongated crustacean zooplankton (other euphausiids, shrimp), both experimentally constrained (by tethering or encagement, e.g., Greenlaw, 1977; Kristensen and Dalen, 1986; Everson et al., 1990; Foote, 1990; Foote et al., 1990; Wiebe et al., 1990; Demer and Martin, 1995) and in situ (e.g., Hewitt and Demer, 1991, 1996). To obtain target strength information over a wide range of frequencies simultaneously, as well as to elucidate the frequency dependence of the scattering from elongated crustacean zooplankton, broadband insonifications have been made of tethered decapod shrimp (Palaemonetes vulgaris) as well as a species of euphausiid (Meganyctiphanes norvegica) found in the Northwest Atlantic (Chu et al., 1992; Stanton et al., 1994a, 1996) using a broadband chirp sonar. These single-frequency and broadband measurements have been used to develop and corroborate empirical and theoretical scattering models.

Empirical models (e.g., Greene et al., 1991) have relied on relating a single parameter (e.g., zooplankton size or wet weight) to acoustic target strength through a simple regression relationship. Initial theoretical scattering models for zooplankton (including elongated crustacean zooplankton) were based on the Anderson (1950) fluid sphere model (e.g., Greenlaw, 1977, 1979; Johnson, 1977; Penrose and Kaye, 1979), which accounted for animal size and material properties. The first scattering model to consider the elongate and deformable morphology of some of the crustacean zooplankton was developed by Stanton (1988a,b, 1989a,b) to describe the scattering of sound by arbitrarily deformed cylinders of finite length. It became widely recognized that in addition to animal size and shape, animal orientation could have significant effects on the scattering from these elongated plankton (Greenlaw, 1977; Sameoto, 1980; Samovol'kin, 1980; Everson, 1982; Kristensen and Dalen, 1986; Chu et al., 1993).
The theoretical models were further developed and refined (Stanton et al., 1993a,b, 1994a,b, 1996), and this orientational dependence was incorporated by describing scattering from these elongated zooplankton at normal incidence and at a distribution of orientations near broadside incidence using an approximate ray summation model (which takes advantage of the fact that many crustacean zooplankton behave acoustically as weakly scattering bent fluid cylinders). A more precise alternative to these ray models was developed for weakly scattering elongated zooplankton of arbitrary shape at all angles of orientation using a formulation based on the distorted wave Born approximation (DWBA) volume integral (Chu et al., 1993; Stanton et al., 1993b, 1998a,b). In fact, Chu et al. (1993) were able to predict observed echo levels for Antarctic krill at two discrete frequencies (38 and $120 \mathrm{kHz}$ ) using the DWBA model implemented with certain theoretical orientation distributions, but due to the absence of photographic measurements, were unable to verify actual krill orientation.

This paper summarizes an analysis of the effect of animal orientation on acoustic scattering by Antarctic krill. Both single-frequency and broadband acoustic scattering measurements were made of individual krill, and each animal was filmed during insonification with a high-magnification underwater video system. A separate paper (McGehee et al., in press) is devoted to investigating the orientation-dependence of the single-frequency $(120 \mathrm{kHz})$ target strength measurements. The work summarized herein is focused on interpretation of the broadband $(500-\mathrm{kHz}$ center frequency) scattering measurements in light of orientational information extracted using a novel video analysis technique. By coupling the collected broadband echo spectra with orientation data from the video footage, the effect of animal orientation on the frequency-dependent scattering characteristics of elongated, fluid-like zooplankton such as krill may be elucidated. The echo spectra collected from krill in different orientations are presented. These echoes are compared to the theoretical model results of the DWBA volume integral, which predicts echo spectra for all angles of orientation. Subsequently, a classification inversion using the modelbased Covariance Mean Variance Classification (CMVC) technique (Martin Traykovski et al., 1998) is carried out. Both theoretical and empirical models are employed to invert the echo spectra backscattered from the krill for angle of orientation; that is, the CMVC inversion technique uses these models to predict angle of orientation during each insonification based on the received echo spectrum.

\section{METHODS}

\section{A. Scattering experiment}

Acoustic and video data were collected during a weeklong experiment (17 August-21 August 1995) at the Long Marine Laboratory of the University of California at Santa Cruz (UCSC). Antarctic krill (Euphausia superba) had been captured in the Southern Ocean near Palmer Station, Antarctica in February 1995, and placed in individual containers without food for long-term storage under refrigeration at the University of California at Santa Barbara, until transport to 


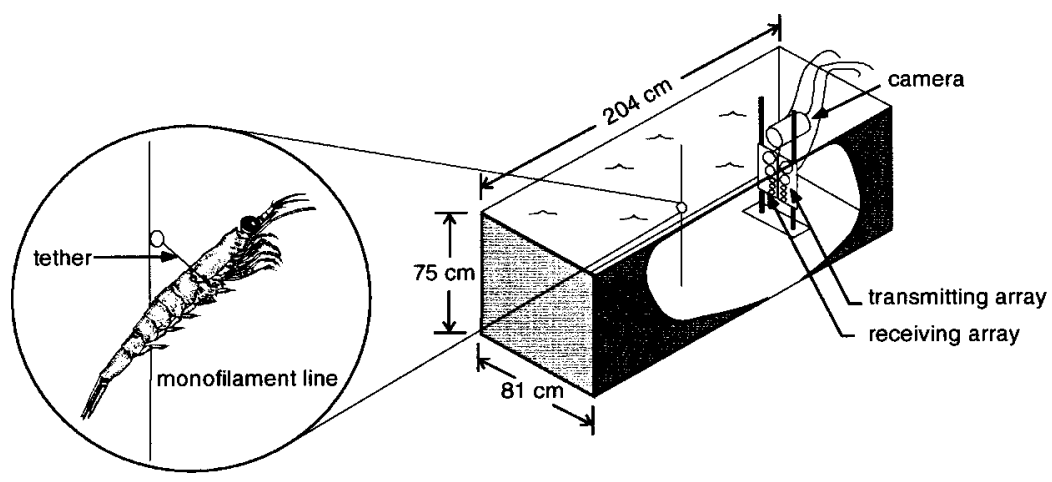

FIG. 1. Scattering experiments were carried out in a portion of a modified fiberglass dolphin transporter. To permit freedom of movement during insonification, the animals were tethered to a vertical section of monofilament line with an acoustically transparent fine strand around the abdomen. The tethered animal was lowered to the focal point of a side-looking transducer pair. A high-magnification underwater video camera was mounted above the transducer array, looking slightly downward at the animal. Each insonification was marked with an acoustic pulse on the audio track of the video tape, so that animal orientation at the time of insonification could be determined.

UCSC in August. Acoustic experiments included insonification of each live animal with a $200-\mu$ s duration broadband chirp of center frequency $500 \mathrm{kHz}(\sim 350-750 \mathrm{kHz})$.

Insonifications of the krill were made with a pulse-echo acoustic data acquisition system. The transmit/receive transducer pair was mounted in a side-looking transducer bank, and the animal was positioned at the focal point of the transducer pair at a range of $51 \mathrm{~cm}$ (Fig. 1). This bistatic experimental setup is similar to that described by Stanton (1990), and has been used extensively (e.g., Chu et al., 1992; Stanton et al., 1993b, 1994a,b, 1996) to make scattering measurements of zooplankton. During insonification, individual krill were suspended one at a time in a $2-\mathrm{m}$ long by $0.8-\mathrm{m}$ wide by $0.75-\mathrm{m}$ deep tank (adapted from a fiberglass dolphin transporter) filled with filtered, chilled seawater (maintained between 2 and $5{ }^{\circ} \mathrm{C}$ ). Each animal was tethered to a vertically suspended $0.12-\mathrm{mm}$ monofilament line with a fine (55 $\mu \mathrm{m})$, acoustically transparent synthetic strand tied around the first or second abdominal segment. This short tether allowed freedom of movement during insonification, while constraining the animal to remain within $1 \mathrm{~cm}$ of the focal point of the transducer pair. Target strength (TS) measurements made of these moving krill using the $500-\mathrm{kHz}$ broadband transducer in this bistatic configuration are estimated to be within 0.5 $\mathrm{dB}($ at $350 \mathrm{kHz})$ and $0.6 \mathrm{~dB}$ (at $750 \mathrm{kHz}$ ) of the actual values. Error estimates were based on both systematic and ran- dom sources of acoustic measurement error, including transducer misalignment and animal positional variability. To allow investigation of the relationship between the acoustic scattering of an organism and its orientation, each animal was filmed during insonification with a high-magnification underwater video system. The video camera was mounted directly above and slightly behind the transducer bank, looking across and slightly downward at the animal. Each insonification was marked with an acoustic pulse recorded on one audio track of the Hi-8 video tape, while a time code was continuously recorded on the other audio track. This allowed direct correlation between each acoustic return and the orientation of the animal at the time of insonification (to within $1 / 30 \mathrm{~s})$. The freedom of movement permitted by the tether allowed the animal to assume a wide range of positions relative to the transducer, including lateral, dorsal, ventral, and end-on aspects.

After insonification, several measurements were made of each animal, including animal length, carapace height, carapace width, telson (central lobe of tail) length, and wet weight (Table I). If the telson was broken, total length (L2) for that animal was estimated by scaling the length (L1) to the end of the uropods (lateral lobes of tail) according to the ratio (L2/L1) observed in other individuals of similar size; telson length was also estimated by comparison to other animals of similar dimensions. Excess water was removed and

TABLE I. Summary of measurements made of Antarctic krill. Total length measured from center of eye to tip of telson; carapace height measured at maximum dorso-ventral width; carapace width measured at maximum lateral width. The asterisk indicates broken telson: for these animals, total length (L2) was estimated by scaling length to end of uropods (L1) according to the ratio (L2/L1) observed in other individuals of similar size; telson length also estimated by comparison to other animals of similar dimensions. Video analysis not possible for animals 12-14 due to blurring of the video images caused by condensation on the inside of the camera housing. Dimensions in $\mathrm{mm}$, weights in $\mathrm{g}$.

\begin{tabular}{|c|c|c|c|c|c|c|c|c|}
\hline Animal No. & $\begin{array}{l}\text { Total } \\
\text { length }\end{array}$ & $\begin{array}{c}\text { Carapace } \\
\text { height }\end{array}$ & $\begin{array}{l}\text { Carapace } \\
\text { width }\end{array}$ & $\begin{array}{l}\text { Telson } \\
\text { length }\end{array}$ & $\begin{array}{c}\text { Wet } \\
\text { weight }\end{array}$ & $\begin{array}{c}\text { Dry } \\
\text { weight }\end{array}$ & $\begin{array}{c}\text { No. of echoes } \\
\text { collected }\end{array}$ & $\begin{array}{c}\text { No. of video images } \\
\text { analyzed }\end{array}$ \\
\hline 01 & 37.6 & 5.5 & $\ldots$ & 7.4 & 0.38 & 0.0872 & 1000 & 1000 \\
\hline 02 & 42.2 & 5.9 & $\ldots$ & 8.6 & 0.51 & 0.1111 & 1000 & 400 \\
\hline 03 & 41.4 & 5.4 & 4.4 & 8.4 & 0.55 & 0.1153 & 1000 & 400 \\
\hline 04 & 38.9 & 4.8 & 4.35 & 7.7 & 0.38 & 0.0932 & 1000 & 400 \\
\hline 05 & 41.4 & 5.3 & 4.4 & 7.1 & 0.56 & 0.1181 & 1000 & 1000 \\
\hline 06 & 40.15 & 4.9 & 4.2 & 6.25 & 0.44 & 0.0916 & 1000 & 200 \\
\hline $07^{*}$ & 33.3 & 4.4 & 3.65 & 6.2 & 0.24 & 0.0532 & 1000 & 1000 \\
\hline 08 & 29.75 & 3.95 & 3.2 & 6.0 & 0.16 & 0.0363 & 1000 & 200 \\
\hline 09 & 37.6 & 5.25 & 4.45 & 6.35 & 0.36 & 0.0702 & 1000 & 200 \\
\hline 10 & 37.3 & 4.95 & 4.0 & 7.1 & 0.36 & 0.0689 & 1000 & 200 \\
\hline 11 & 40.6 & 5.4 & 4.15 & 7.85 & 0.44 & 0.1016 & 1000 & 200 \\
\hline 12 & 39.6 & 5.4 & 4.0 & 7.35 & 0.44 & 0.0884 & 1000 & none \\
\hline $13^{*}$ & 42.9 & 6.0 & 4.9 & 8.1 & 0.55 & 0.1285 & 1000 & none \\
\hline 14 & 42.45 & 6.15 & 4.6 & 7.7 & 0.58 & 0.1265 & 1000 & none \\
\hline
\end{tabular}


each organism was frozen; dry weight was measured after oven-drying at a later date. During the experiment, 14 individuals were insonified and the return echoes from 1000 acoustic transmissions were collected from each animal.

\section{B. Acoustic data processing}

To obtain the echo spectrum representing the actual acoustic return from the animal, the raw signals received from the krill were combined with the results of calibration measurements, taken at the beginning (16 August 1995) and again at the end (24 August 1995) of the scattering experiments. During calibration, the transmit and receive transducers were aimed at each other with no target in the beam, and a calibration signal was transmitted. The transmitted and received voltage time series were collected for these calibration measurements [subscript $C$ in Eq. (1)]. During the scattering experiments, the transducers were aimed forward, focused at a range of about half a meter, and the animal was placed in this focal region. The transmitted and received voltage time series were collected for the scattering measurements [subscript $S$ in Eq. (1)], and for each received acoustic return the calibrated echo spectrum was computed as:

$$
\left|f_{\mathrm{bs}}\right|=\left(\frac{V \mathrm{rec}_{S}}{V \operatorname{rec}_{C}}\right) \cdot\left(\frac{V \mathrm{xmit}_{C}}{V \mathrm{xmit}_{S}}\right) \cdot\left(\frac{r_{S}^{2}}{r_{C}}\right) .
$$

In Eq. (1), $f_{\text {bs }}$ is the acoustic backscattering amplitude of the animal, and is a measure of the efficiency with which an object scatters sound back toward the sound source $\left[f_{\mathrm{bs}}\right.$ is related to $\sigma_{\mathrm{bs}}$, the differential backscattering cross section (Clay and Medwin, 1977), by $\left.\sigma_{\mathrm{bs}}=\left|f_{\mathrm{bs}}\right|^{2}\right] . \quad V \operatorname{rec}_{C}$ and $V$ xmit $_{C}$ were computed by taking the absolute value of the FFT of the received and transmitted voltage time series for calibration. $V \mathrm{xmit}_{S}$ was computed as the absolute value of the FFT of the transmitted voltage time series for scattering measured at the end of each run (every 200 pings). To compute $V \operatorname{rec}_{S}$, a fixed rectangular window was applied to the received voltage time series for each return (to capture only the echo from the animal) before applying the FFT. The scattering and calibration distances were $r_{S}=50.9 \mathrm{~cm}$ and $r_{C}=57.8 \mathrm{~cm}$, respectively. The echo spectrum (TS $\left.=20 \log \left|f_{\mathrm{bs}}\right|\right)$ for each return was then sampled at 203 points between 348.33 and $685 \mathrm{kHz}$ (due to undesirable transducer frequency response characteristics in the upper end of its frequency range, the full bandwidth $(\sim 350-750 \mathrm{kHz})$ of the collected data was not used). It is this sampled echo spectrum that was used in analyzing the effects of orientation on the frequency-dependent scattering characteristics of Antarctic krill.

\section{Video data processing}

Video images for 11 of the 14 animals were analyzed to extract the angle of orientation of the krill corresponding to each echo spectrum (Table I). For some animals, orientation was extracted from only a subset of the 1000 images, since the video analysis procedure was extremely time-consuming. Condensation inside the video camera housing obscured the images for Animals 12-14, so that video analysis was not possible for these individuals.

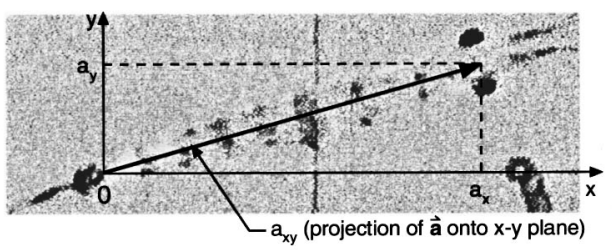

B.

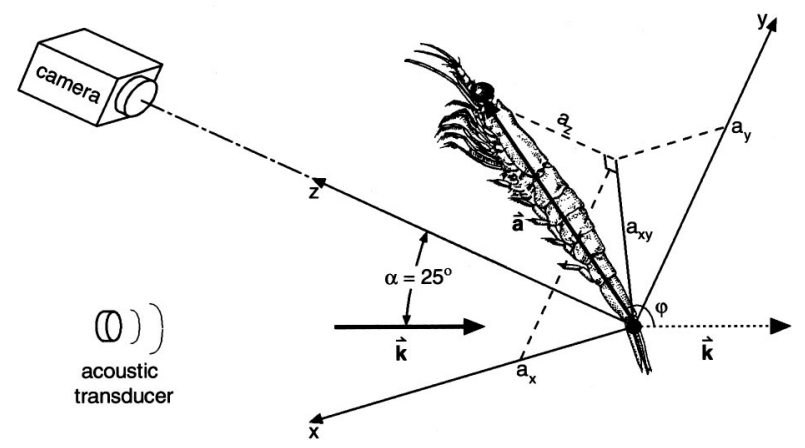

FIG. 2. Geometry for determination of angle of orientation $\varphi$ from the video images. (A) Image gives the projection of the animal vector $\overrightarrow{\mathbf{a}}$ onto the $x-y$ plane of a three-dimensional (3-D) coordinate system with origin situated at the base of the krill's telson. $a_{x}$ and $a_{y}$ computed directly from measurements made of the image; (B) 3-D sketch: $a_{z}$ determined from geometry; $\varphi$ computed using Eq. (2); incident acoustic wave vector $\mathbf{k}$ is in the $y-z$ plane, at an angle of $\alpha=25^{\circ}$ relative to the camera line of sight.

To determine the orientation of the animal corresponding to each received echo spectrum, it was necessary to extract from the video tape only the frames captured at the time of each insonification, as indicated by the acoustic pulses recorded on the audio track. To accomplish this, the audio and video channels of the original video tapes were duplicated, and a screen-burn of the time code was made on the duplicates to allow easier identification and location of frames of interest. The tapes were advanced frame-by-frame using a video editing deck which permitted frame-by-frame advancement with an audible audio track, and the time code of each frame in which an acoustic pulse occurred was noted. The video frames of interest were then identified upon playback by the screen-burned time code, captured, digitized, and stored in TIFF format using the public domain image processing and analysis program NIH Image for the Macintosh.

For each image, the origin of a three-dimensional rectilinear coordinate system was situated at the base of the animal's telson [Fig. 2(A)]. In this coordinate system, the video image represents the projection of the animal onto the $x-y$ plane, as the camera looks in the negative $z$ direction. The animal itself is then represented by a vector $\overrightarrow{\mathbf{a}}$ from the origin to the point midway between the center of the eyes. Measurements were made to determine the $x$ and $y$ coordinates of $\overrightarrow{\mathbf{a}}\left(a_{x}\right.$ and $\left.a_{y}\right)$ for each image. Using a custom-built Matlab ${ }^{\circledR}$ measurement program, the location of the midpoint between the center of the eyes as well as the anterior edge of the conspicuous dark patch which marks the base of the telson were determined by clicking these points with a mouse; the colormap was adjusted to facilitate discrimination of these points on the images. The projected length of the animal $a_{x y}$ 
(in the $x-y$ plane) was computed as $a_{x y}=\sqrt{a_{x}^{2}+a_{y}^{2}}$ directly from these measurements. The $z$ coordinate $a_{z}$ of a was determined using the fact that $a_{z}=\sqrt{|\overrightarrow{\mathbf{a}}|^{2}-a_{x y}^{2}}$ [Fig. 2(B)], where $|\overrightarrow{\mathbf{a}}|$ is the length of $\overrightarrow{\mathbf{a}}$, as measured from an image in which the animal was estimated to be broadside to the camera (i.e., perpendicular to the camera line of sight, $a_{z}=0$ ). The sign of $a_{z}$ was determined by noting whether the animal was head towards ( $a_{z}$ positive), broadside to $\left(a_{z} 0\right)$, or head away from ( $a_{z}$ negative) the camera, as reflected by changes in the projected length of the animal $a_{x y}$ for a succession of single images ( $a_{x y}$ attained its maximum $|\overrightarrow{\mathbf{a}}|$ when the animal was broadside to the camera). This was corroborated by watching the video in real time to estimate when broadside crossings occurred, and noting the head orientation before and after each crossing.

The angle of orientation of the animal was then determined for each image by computing the angle $\varphi$ between the animal vector $\overrightarrow{\mathbf{a}}$ and the incident acoustic wave vector $\overrightarrow{\mathbf{k}}$ [Fig. 2(B)]:

$$
\varphi=\arccos \left(\frac{\overrightarrow{\mathbf{k}} \cdot \overrightarrow{\mathbf{a}}}{|\overrightarrow{\mathbf{k}}||\overrightarrow{\mathbf{a}}|}\right),
$$

where $\overrightarrow{\mathbf{k}} \cdot \overrightarrow{\mathbf{a}}$ is the inner product (dot product) of the two vectors; Eq. (2) follows directly from the definition of the inner product: $\mathbf{k} \cdot \overrightarrow{\mathbf{a}}=|\overrightarrow{\mathbf{k}}||\overrightarrow{\mathbf{a}}| \cos \varphi$, with $0 \leqslant \varphi \leqslant \pi$. For an animal located in the far field of the transducers, the incident acoustic wave is locally planar over the body of the krill, and $\overrightarrow{\mathbf{k}}$ is in the $y-z$ plane (perpendicular to the $x$ axis) of the coordinate system, so that $k_{x}=0$, and $\overrightarrow{\mathbf{k}}=|\overrightarrow{\mathbf{k}}|\left(k_{x}, k_{y}, k_{z}\right)=(2 \pi /$ $\lambda)(0, \sin \alpha,-\cos \alpha)$, where $\lambda$ is the acoustic wavelength and $\alpha$ is the angle between the incident acoustic wave and the camera line of sight. With the animal positioned at the focal point of the transducer pair, this angle was measured as $\alpha=25^{\circ}$ [Fig. 2(B)].

\section{Theoretical modeling}

Acoustic backscattering from a finite-length, arbitrarily shaped, weakly scattering (i.e., having density and sound speed similar to those of the surrounding medium) object in the far field can be described by the general volume integral formulation (Morse and Ingard, 1968) of the distorted wave Born approximation (DWBA):

$$
f_{\mathrm{bs}}=\frac{k_{1}^{2}}{4 \pi} \iiint\left(\gamma_{\kappa}-\gamma_{\rho}\right) e^{i 2\left(\vec{k}_{i}\right)_{2} \cdot \vec{r}_{0}} d \nu_{0}
$$

Recently, Chu et al. (1993) and Stanton et al. (1993b) developed a DWBA model to describe the frequency-dependent scattering characteristics of elongated, fluid-like zooplankton at all angles of orientation. If the body has a circular cross section at every point along its lengthwise axis, the DWBA volume integral [Eq. (3)] may be reduced to a line integral along this axis (Stanton et al., 1998b), yielding an accurate expression for the scattering from an elongated, weakly scattering fluid-like finite cylinder as a function of size, shape, material properties, and angle of orientation:

$$
\begin{aligned}
\mathrm{TS}= & 20 \log \mid \frac{k_{1}}{4} \int_{\vec{r}_{\mathrm{pos}}}\left(\gamma_{\kappa}-\gamma_{\rho}\right) e^{i 2\left(\vec{k}_{i}\right)_{2} \cdot \overrightarrow{\mathrm{r}}_{\mathrm{pos}}} \\
& \times a \frac{J_{1}\left(2 k_{2} a \cos \beta_{\mathrm{tilt}}\right)}{\cos \beta_{\mathrm{tilt}}}\left|d \vec{r}_{\mathrm{pos}}\right| \mid .
\end{aligned}
$$

In Eq. (4), TS $=20 \log \left|f_{\mathrm{bs}}\right|, k=2 \pi / \lambda$ is the acoustic wave number $(\lambda=c / f$ where $c$ is the sound speed in $\mathrm{m} / \mathrm{s}$ and $f$ is the acoustic frequency in $\mathrm{Hz}),\left(\vec{k}_{i}\right)_{2}=\vec{k}_{2}=\vec{k}_{1} / h, J_{1}$ is a Bessel function of the first kind of order $1, \gamma_{p}=\left(\rho_{2}\right.$ $\left.-\rho_{1}\right) / \rho_{2}$, and $\gamma_{\kappa}=\left(\kappa_{2}-\kappa_{1}\right) / \kappa_{1}$ with compressibility $\kappa$ defined as $\kappa_{i}=1 /\left(\rho_{i} c_{i}^{2}\right)$; subscript 1 refers to the surrounding medium (seawater), subscript 2 refers to the fluid-like medium of the zooplankton body, so that $\left(\gamma_{\kappa}-\gamma_{\rho}\right)=\left(1 / g h^{2}\right)$ $+(1 / g)-2$, where $g=\rho_{2} / \rho_{1}$ is the density contrast of the organism relative to water, and $h=c_{2} / c_{1}$ is its sound speed contrast. This model predicts the scattering from a deformed fluid-like cylindrical body of arbitrary shape (i.e., the crosssectional radius of the cylinder $a$ can vary along the lengthwise axis) for any angle of orientation relative to the incident acoustic wave by integrating the scattering contributions of each infinitesimally thin cross section (located at $\vec{r}_{\text {pos }}$ along the lengthwise axis, at an angle $\beta_{\text {tilt }}$ relative to the incident acoustic wave) over of the entire animal body.

The line-integral DWBA formulation in Eq. (4) can be implemented in a numerical integration scheme to model the orientational dependence of the scattering from an animal of known size, shape, and material properties. The animal body may be discretized into several cylindrical cross sections, each defined by a position $\vec{r}_{\text {pos }}$ along the lengthwise axis of the animal body, a radius $a$, a density contrast $g$, and a sound-speed contrast $h$. This discretization was achieved by digitizing the outline of the animal from a video image (in lateral aspect if possible), capturing several points along the dorsal and ventral surfaces [refer to Figs. 4 and 5, parts (A) and (B)]. This outline was then scaled to size using the measurements made of the animal after insonification (Table I), and $a$ and $\vec{r}_{\text {pos }}$ were computed for each discrete segment from each dorso-ventral pair of points. The density contrast and sound-speed contrast were held constant over the animal body; values of $g=1.0357$ and $h=1.0279$ (as measured for Euphausia superba by Foote, 1990) were used. For a particular angle of orientation of the animal, the backscatter at each of 203 acoustic frequencies (between 348.33 and $685 \mathrm{kHz}$ ) was computed as the sum of the scattering contributions of each of the cylindrical cross sections due to an incident acoustic wave vector $\vec{k}_{1}=\left(\left|\vec{k}_{1}\right| \cos \beta_{\text {tilt }},\left|\vec{k}_{1}\right| \sin \beta_{\text {tilt }}\right)$. Since the orientational dependence of the scattering predicted by the DWBA model is symmetrical about $180^{\circ}$ for an arbitrary shape with circular cross section, the model was implemented by varying the angle of orientation in $1^{\circ}$ increments between $0^{\circ}$ and $180^{\circ}$. Appendix A of McGehee et al. (in press) contains Matlab ${ }^{\circledR}$ code to implement this numerical integration scheme.

\section{E. Inversion for angle of orientation}

If the acoustic backscattered energy from elongated, fluid-like zooplankton exhibits a strong orientational depen- 
dence, biomass estimates for krill and other euphausiids based on the interpretation of acoustic survey data would be much improved by in situ determination of angle of orientation. Classification inversion schemes have been developed which can categorize individual zooplankton into distinct scatterer types (e.g., fluid-like, elastic-shelled, gas-bearing), as well as invert for specific parameters (e.g., animal size, animal orientation), based on the signature information contained in the return spectra of broadband insonifications of the animals (Martin et al., 1996; Martin Traykovski et al., 1998). Such a classification inversion approach was applied to the echo spectra collected from these krill to investigate the feasibility of inverting broadband acoustic returns for angle of orientation for these fluid-like zooplankton.

The Covariance Mean Variance Classifiers (CMVC) (Martin Traykovski et al., 1998) are a set of advanced model-based techniques which classify observed echo spectra based on the correspondence between the observations and model predictions. Theoretical or empirical scattering models are used to construct a model space, which consists of model realizations representing predictions of the models for particular parameter values spanning the entire parameter space. For the E. superba data, the objective of this classification is to invert observed echo spectra for a specific parameter value: angle of orientation. The CMVC techniques can be implemented in several alternative configurations, one of which may be employed to search the entire physical model parameter space for the best-match model realizations for a set of observations, and report the parameter values of interest. Determination of the best match is based on the CMV metric $(\mathbf{C})$, which quantifies the correspondence between an ensemble of observed echo spectra (D) and all the model realizations $(\mathbf{M})$. The best-match model realization is determined by maximizing the CMV metric; to achieve a good match between an observation and a model realization, not only must the spectral structure (e.g., the location of peaks and nulls) be similar (as measured by the covariance $\mathbf{K})$, but both the mean echo levels and the spectral variability (e.g., null depth) must also be comparable (quantified by the mean similarity $\mathbf{X}$ and the variance similarity $\mathbf{U}$; Martin Traykovski et al., 1998). The CMV metric (C) is computed as

$$
\mathbf{C}=\operatorname{CMV}(\mathbf{M}, \mathbf{D})=\mathbf{K} \cdot \mathbf{X} \cdot \mathbf{U} .
$$

Note that in Eq. (5), $\mathbf{C}_{i j}=\left(\sum_{k=1}^{n_{p}} \mathbf{D}_{i k}^{T} \mathbf{M}_{k j}\right) \cdot \mathbf{X}_{i j} \cdot \mathbf{U}_{i j}$, since $\mathbf{K}=\mathbf{D}^{T} \mathbf{M}$ is the covariance $\left(0 \leqslant \mathbf{K}_{i j} \leqslant 1\right.$, see Papoulis, 1991) between the observed data matrix $\mathbf{D}$ and the model space matrix $\mathbf{M}$. Each column of $\mathbf{D}$ contains a mean-subtracted, energy-normalized observed echo spectrum, whereas each column of $\mathbf{M}$ contains a mean-subtracted, energy-normalized model realization. $\mathbf{X}$ and $\mathbf{U}$ are the mean and variance similarity matrices $\left(0 \leqslant \mathbf{X}_{i j}, \mathbf{U}_{i j} \leqslant 1\right.$, where $\mathbf{X}_{i j}=1$ indicates that the $i$ th observation and the $j$ th model realization have the same mean echo levels, and $\mathbf{U}_{i j}=1$ indicates that the $i$ th observation and the $j$ th model realization have identical variance), $n_{p}$ is the number of points in each echo spectrum, and the centered $\operatorname{dot}(\cdot)$ indicates element-wise multiplication of matrices. The best-match model realization for the $i$ th observed echo spectrum is found by determining the column $m$ in which the maximum value in row $i$ of $\mathbf{C}$ occurs, for example, for the first observation, $i=1$ and the best-match $m$ is the realization for which $\mathbf{C}_{1 m}=\max \left(\mathbf{C}_{1 j}\right)$ over all $j$. The inversion result for observation $i$ is then the angle of orientation associated with the model realization $m$ that best predicts that observation.

The ability of the classifier to invert echo spectra for angle of orientation depends to a large extent upon the representativity of the model space, that is, whether it accurately predicts the scattering over the entire observed range of the parameter values with sufficient resolution. In this inversion of krill echo spectra for angle of orientation, two types of theoretical model spaces and two types of empirical model spaces were employed. The theoretical model spaces, consisting of model realizations generated from predictions of the DWBA model [Eq. (4)], include a single, sizeconstrained generic model space for all animals, and 11 animal-specific model spaces, one for each animal. The generic theoretical model space was based on several distinct discretizations of a euphausiid body, digitized from selected images to represent different animal shapes. Model predictions were made as described in the previous section, using each shape (scaled to approximately the size of the animal) as input into the DWBA. An animal-specific theoretical model space was generated for each of the 11 animals; the discretized shape used as input into the DWBA model was digitized from an image of the particular animal, so that it corresponded with the exact size and shape of that krill. The empirical model spaces included both a single generic empirical model space, constructed by interpolating the echo spectra received from Animal 01 over angle of orientation (with $1^{\circ}$ resolution), and animal-specific empirical model spaces for Animals 01, 03, 05, and 09, generated by interpolating the empirical data in the same manner, but based on the observed echo spectra for that particular animal. Only these four animals exhibited a sufficiently wide range of orientations to generate a model with nearly complete angular coverage (see orientation distribution histograms in Fig. 3). For all 11 animals, inversions based on the generic theoretical model space, the appropriate animal-specific theoretical model space, and the generic empirical model space were carried out. Inversions based on the appropriate animalspecific empirical model space were performed for Animals 01, 03, 05, and 09 only.

\section{RESULTS AND DISCUSSION}

\section{A. Variability in echo spectra with angle of orientation}

Acoustic returns from the krill varied considerably with angle of orientation (Fig. 3). Echo spectra from animals near broadside incidence relative to the incident acoustic wave $\left(\varphi=90^{\circ}\right)$ were characterized by widely spaced deep nulls (often $20 \mathrm{~dB}$ or greater), usually separated by $200 \mathrm{kHz}$, whereas the frequency response of off-broadside echoes exhibited a more erratic structure, with several closely spaced $(<50$ $\mathrm{kHz}$ ) nulls of variable depth. The scattering from elongated, fluidlike zooplankton at broadside incidence is thought to be dominated by the constructive and destructive interference 


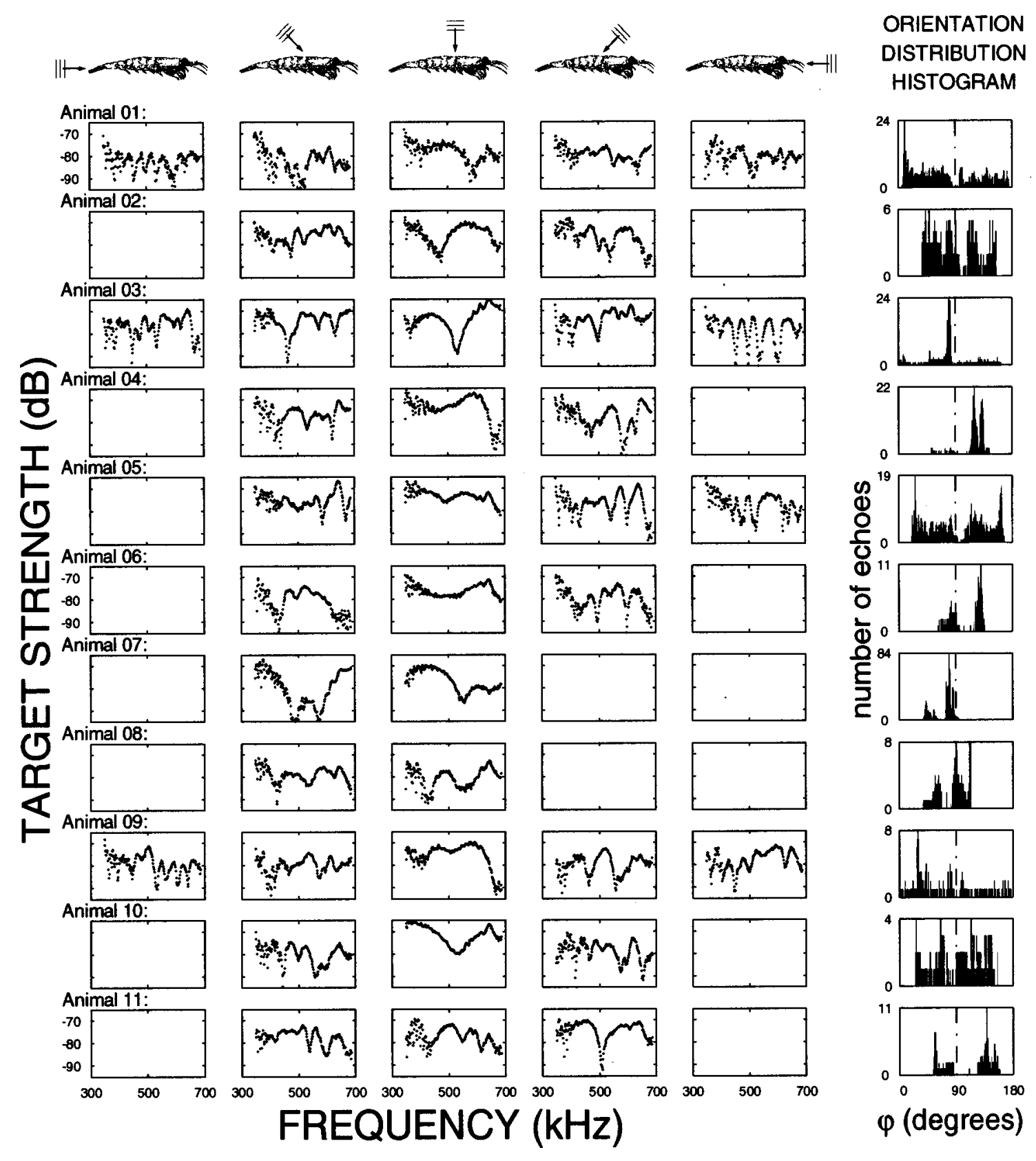

FIG. 3. Examples of echo spectra received from 11 krill (Animals $01-11$, rows) at five angles of orientation, with acoustic wave incident at $\varphi \cong 0^{\circ}, 45^{\circ}, 90^{\circ}$, $135^{\circ}, 180^{\circ}\left( \pm 15^{\circ}\right)$ (first five columns); empty plots indicate that the animal did not assume that orientation during the experiment. A histogram of the orientation distribution for each animal during insonification is shown at right.

between the echo from the front interface and the echo from the back interface of the animal (see Fig. 8, CASE 1). At off-broadside angles, contributions from other scattering features of the animal body are believed to become more significant, resulting in a more complicated/erratic interference pattern with many nulls (Stanton et al., 1994b, 1998b).

The effect of orientation on average echo levels was investigated by computing the mean target strength over all frequencies ( $\left.\overline{\mathrm{TS}}=10 \log \mid \overline{\sigma_{\mathrm{bs}}}\right)$ of the echo spectrum received at each angle of orientation $\varphi$. These spectrally averaged TSs (not shown) were found to be about $5 \mathrm{~dB}$ higher near broadside incidence versus off-broadside for most animals. For the larger animals (e.g., Animals 02, 03, 05, 06, 11), average target strengths over the frequency band were approximately
$-70 \mathrm{~dB}$ at orientations near broadside versus $-75 \mathrm{~dB}$ offbroadside, whereas for the smaller animals (e.g., Animals 01, $08,09,10)$, the average TS near-broadside incidence was about $-75 \mathrm{~dB}$, versus off-broadside spectrally averaged target strengths of approximately $-80 \mathrm{~dB}$. These observations are consistent with what is expected based on the physics of scattering from elongated objects (Stanton, 1988a,b, 1989a, 1993a,b) since at broadside incidence, an elongated krill presents a much larger backscattering cross section to the incident acoustic wave than at any other angle of orientation.

There is a maximum of $\pm 15^{\circ}$ uncertainty in the calculation of $\varphi$ using the video analysis method described to extract angle of orientation from two-dimensional images of the animal. Some of this uncertainty arises from possible 
A.

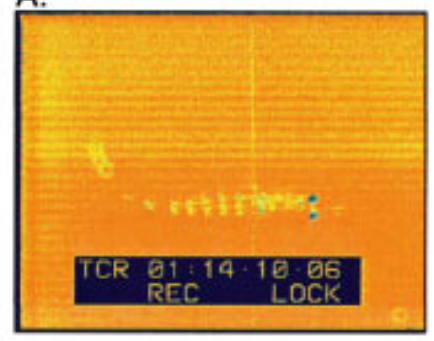

image b01r1088.tif

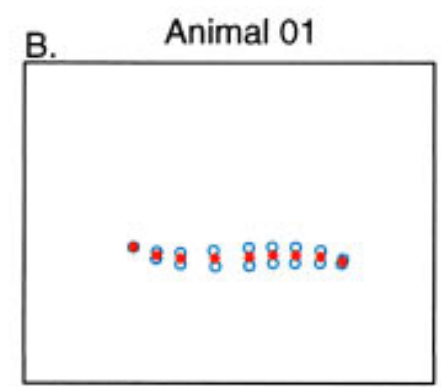

shape used for DWBA model

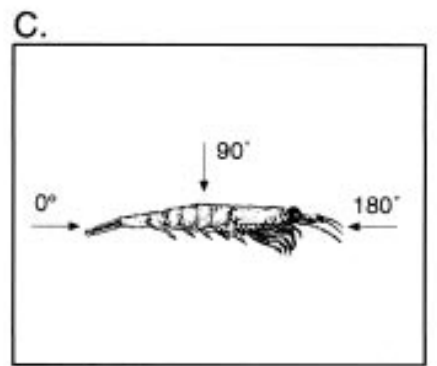

orientation reference
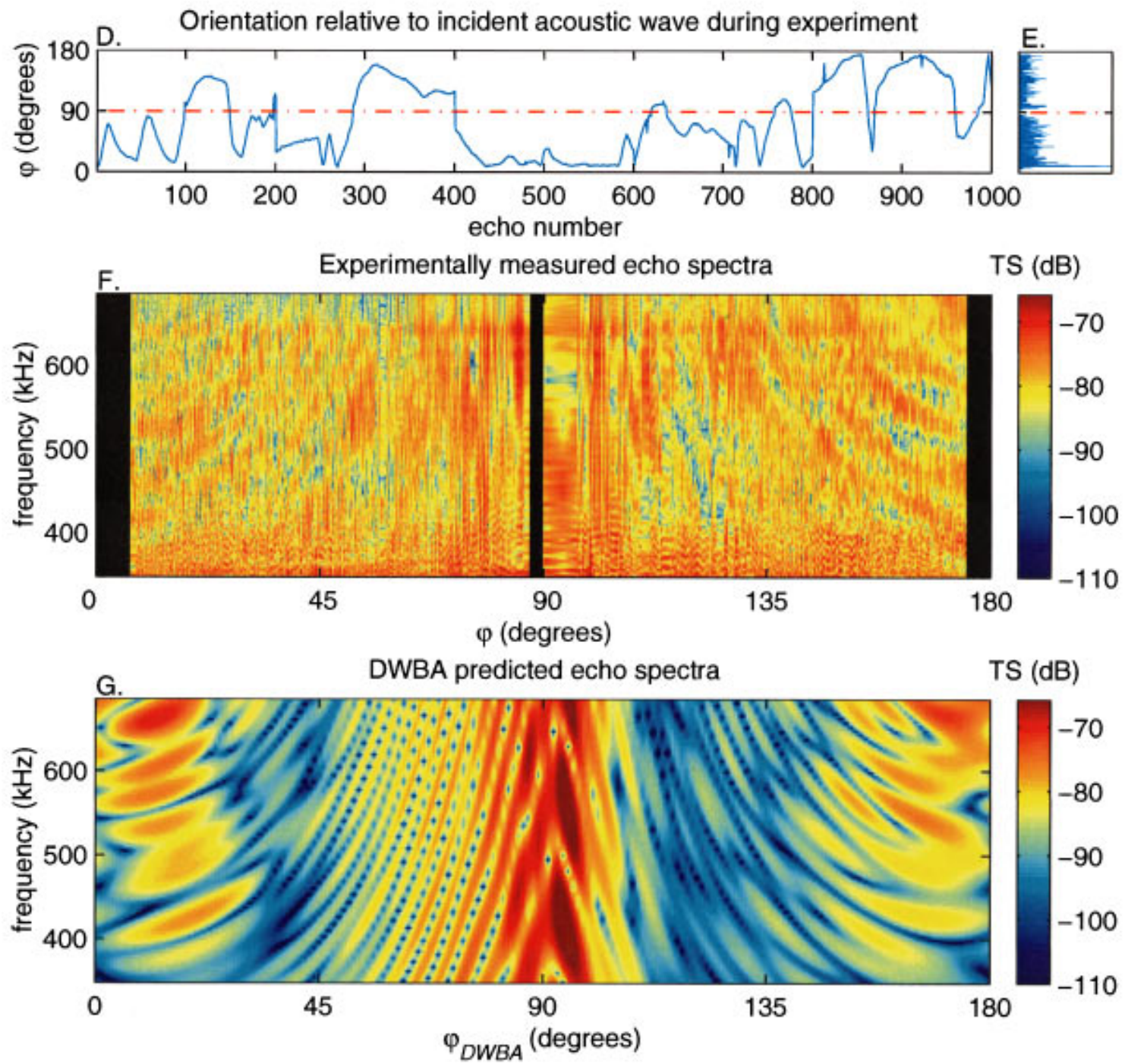

FIG. 4. Experimentally measured echo spectra versus DWBA model predictions for Animal 01: (A) video image used to digitize animal shape; (B) discretization of animal body; (C) orientation reference for DWBA modeling; (D) time series of $\varphi$ during insonification; (E) orientation distribution histogram $(n=1000)$; (F) measured echo spectra ( $y$ axis, TS in color) versus angle of orientation $\varphi$ ( $x$ axis, interpolation over more than $1.5^{\circ}$ is blacked out); $(\mathrm{G})$ echo spectra predicted by DWBA model.

error during measurement of the video frames, as a result of the limited pixel resolution of the image and the curvature of the animal body, both of which can affect the measured projected length of the animal. Measurement error is greatest when the animal is broadside to the camera and decreases non-linearly at angles off-broadside, since very small changes (errors) in the measured length when the projected length is maximum result in greater changes in computed angle relative to measurement errors made when the animal is off-broadside relative to the camera. An additional source of error in the calculation of $\varphi$ arises from small changes in the value of $\alpha$ [the angle between the camera line of sight and the transducer line of sight, see Fig. 2(B)] due to uncertainty in the exact fore-aft camera position relative to the 


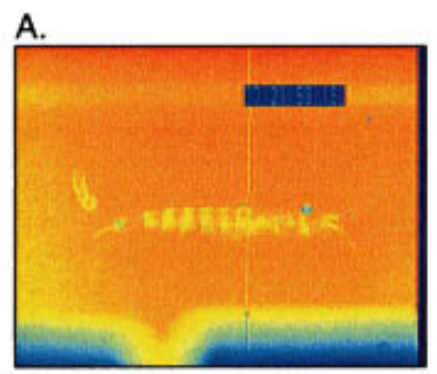

image b03r0107.tif
B. Animal 03

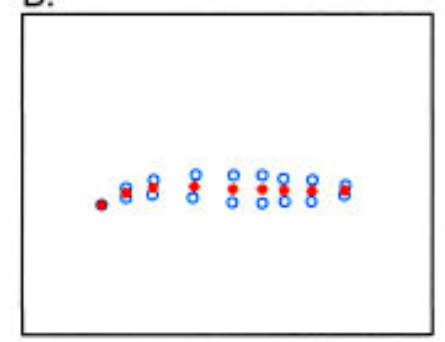

shape used for DWBA model

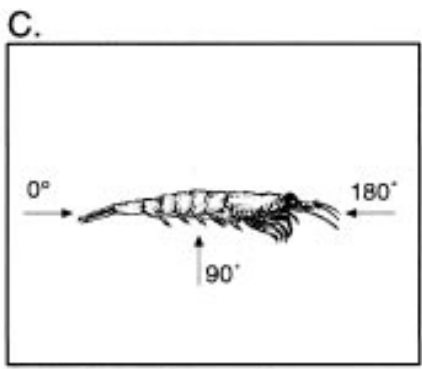

orientation reference
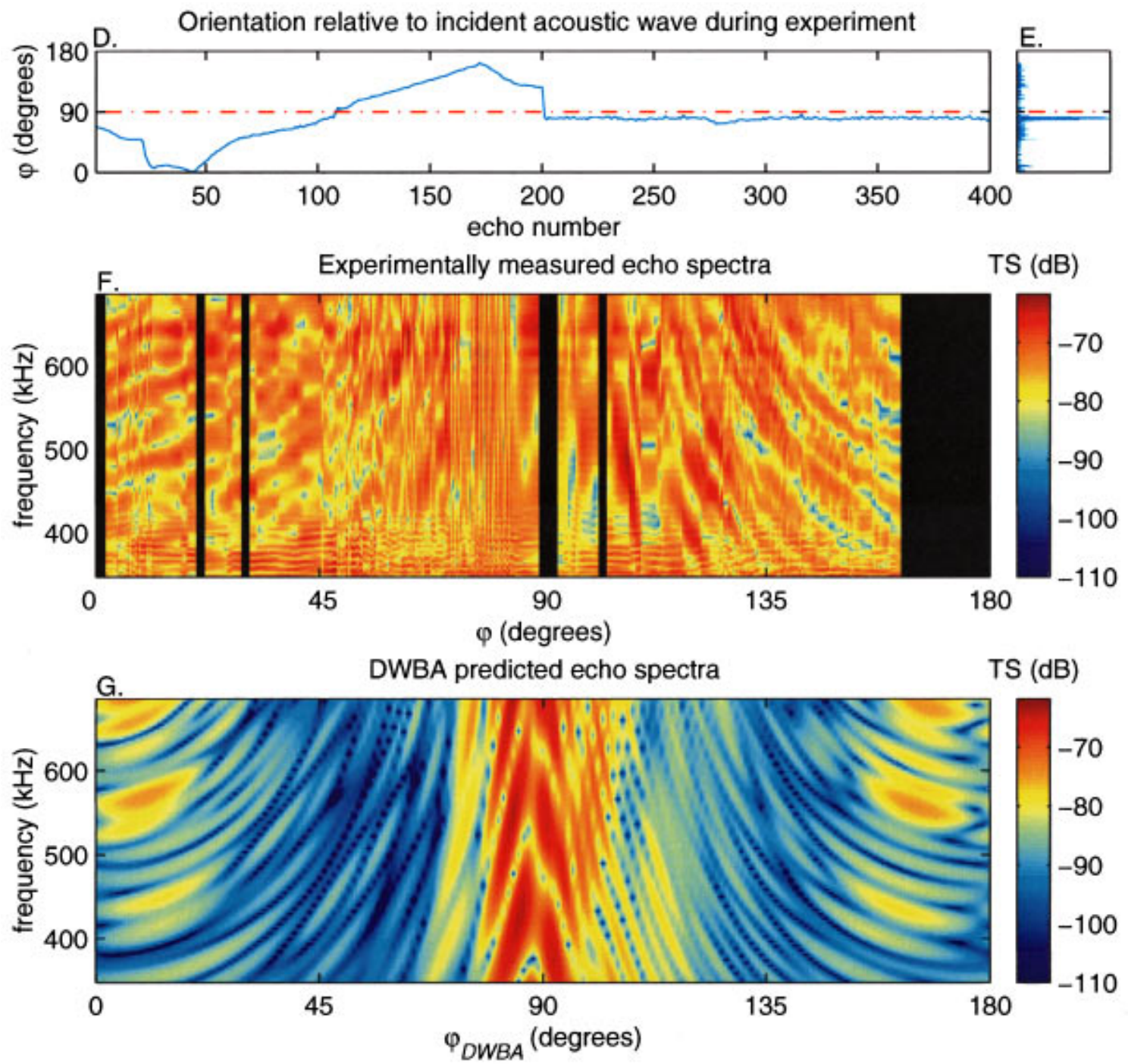

FIG. 5. Experimentally measured echo spectra versus DWBA model predictions for Animal 03: (A) video image used to digitize animal shape; (B) discretization of animal body; (C) orientation reference for DWBA modeling; (D) time series of $\varphi$ during insonification; (E) orientation distribution histogram $(n=400)$; $(\mathrm{F})$ measured echo spectra ( $y$ axis, TS in color) versus angle of orientation $\varphi\left(x\right.$ axis, interpolation over more than $1.5^{\circ}$ is blacked out); $(\mathrm{G})$ echo spectra predicted by the DWBA model.

transducer bank. The maximum uncertainty of $\pm 15^{\circ}$ is a conservative estimate based on a sensitivity analysis of the effect of these sources of error on the calculated values of $\varphi$. The single-camera video system used to record animal orientation cannot provide the same accuracy as that obtainable by measuring animal orientation with a two-camera or a stereo-camera system. Future measurements would benefit from the implementation of a more sophisticated camera sys- tem. In the experiment, not all animals assumed all orientations during insonification, as is evident from the histograms of the orientation distribution measured for each animal (Fig. 3); Animals 01, 03, 05, and 09 were insonified at the widest range of angles $\varphi$. As a result of the extreme sensitivity of the measurement technique to very small changes in projected length near broadside, many of the histograms exhibit low echo counts at or near $\varphi=90^{\circ}$. 


\section{B. Comparison to DWBA model theoretical predictions}

Experimentally measured echo spectra versus angle of orientation $\varphi$ were compared to theoretical model predictions based on a DWBA model for each animal. Two examples are presented: Animal 01 (Fig. 4) and Animal 03 (Fig. 5); results for these animals are shown because they assumed the widest range of orientations during insonification, providing the best angular coverage over which to visualize the comparison between observations and theory. The pattern of changes in echo spectra with $\varphi$ is very similar for the experimentally measured data and the DWBA model predicted spectra for all 11 krill, with only one or two nulls apparent in the frequency responses near broadside incidence, whereas the spectra become much more oscillatory (more peaks and nulls) off-broadside. Although the patterns agree qualitatively, the DWBA model predicts a much greater drop in TS (about $20 \mathrm{~dB}$ ) as orientation changes from broadside incidence to off-broadside (i.e., $20^{\circ}-60^{\circ}$ and $120^{\circ}-160^{\circ}$ ) than was actually observed for these animals as they changed orientation [about a 5-dB drop going from broadside incidence to off-broadside; all values were well above the noise floor $(-100$ to $-110 \mathrm{~dB})]$. Another feature of the theoretical model predictions not observed in the data is an increase in echo-levels closer to end-on (head-on and tail-on). In fact, the DWBA model consistently under-predicted off-broadside echo levels for all 11 animals, but model predictions of TS values near end-on were more consistent with the observations.

The DWBA theoretical model, which predicts scattering based on a highly simplified outline of the animal body by approximating it as a deformed cylinder, does not account for contributions of other scattering features of the complex animal body (e.g., rapidly moving legs). These unaccounted for scattering features appear to make significant contributions to the observed echo levels at off-broadside angles of incidence for these krill. Examination of the time series of angle of orientation throughout the experiment revealed that for some animals orientation was rapidly varying [e.g., Animal 01 (see Fig. 4(D)), 02, and 05], whereas other krill changed orientation slowly [e.g., Animal 03 (see Fig. 5(D)), 09, and 10], and still others (especially Animal 07) remained close to the same orientation throughout the experiment. Constraining the krill by tethering around the midsection likely affects their activity level by eliciting an escape response. The impact of animal activity on echo levels is unknown, although some correlation between rapid swimming and elevated echo levels has been observed by these authors and others.

\section{Inversion for angle of orientation}

The information contained in the broadband echo spectra collected from the krill was used to invert the acoustic returns for animal angle of orientation. To accomplish this, a classification inversion using the Covariance Mean Variance Classification approach was performed on the krill echo spectra. This model-based inversion was applied using both theoretical and empirical model spaces (Fig. 6 shows raw and bin-averaged Animal 03 inversion results for both theoretical and empirical model spaces). The raw inversion results tended to be quite variable, particularly for the theoretical model spaces, whereas the bin-averaged results (obtained by averaging over five nearest neighbors) were less sensitive to outliers, and more clearly delineated how each model space performed in inverting for angle of orientation. Surprisingly, inversions obtained using the animal-specific DWBA model space were no more accurate overall than could be achieved with the size-constrained generic DWBA model space (Table II). This indicates that the frequency response of the acoustic return, although sensitive to animal size, is relatively insensitive to animal shape; the animalspecific model space was generated using the digitized shape of each animal scaled to the exact measured size, whereas the generic DWBA model space, although constrained to be approximately the same size as the animal, was generated using several arbitrary shapes digitized from different euphausiid zooplankton. Although the generic DWBA theoretical model space was not able to accurately invert for angle of orientation for many of these animals, this generic theoretical model space has been shown to be quite powerful in discriminating between different types of scatterers; the generic DWBA model has been employed in conjunction with two other theoretical model spaces to classify several different species of zooplankton into three scattering classes (i.e., elastic-shelled, fluid-like, gas-bearing) based on broadband echo spectra (Martin Traykovski et al., 1998). While the resolution of the generic DWBA theoretical model space is sufficient for identifying krill and other euphausiids as elongated, fluid-like scatterers, it is possible that modeling these animals as simple deformed cylinders is not adequate if the goal is to invert single broadband echoes for a particular parameter (e.g., angle of orientation).

Comparison of the inversion results achieved using the different model spaces reveals that a generic empirical model space based on acoustic returns collected from an arbitrarily chosen krill (in this case, Animal 01) may be better able to invert for angle of orientation than an animal-specific theoretical model space, which predicts the scattering with the DWBA model based on the actual size and shape of that particular animal (Table II). For 8 of the 11 krill, the animalspecific DWBA model space did not perform as well as the generic empirical model space [animal-specific DWBA: $36 \%$ correct inversions (s.d. 8.0); generic empirical: 68\% correct (s.d. 8.2), based on an overall average $(n=8)$; correct inversions are those within $\pm 15^{\circ}$ of observed values]. Although the generic empirical model space was based on data collected from an animal of different size and shape, it did account for contributions of other scattering features of the complex animal body not included in the simplifying theoretical model. Orientations predicted using the animalspecific empirical model space were the most accurate overall for Animals 01, 03, 05, and 09, providing a more robust inversion than that achieved with the generic empirical model space (Table II, Fig. 7).

For these inversions (which are based on choosing the global maximum best-match echo spectra), symmetry about broadside incidence was assumed since both the observations 

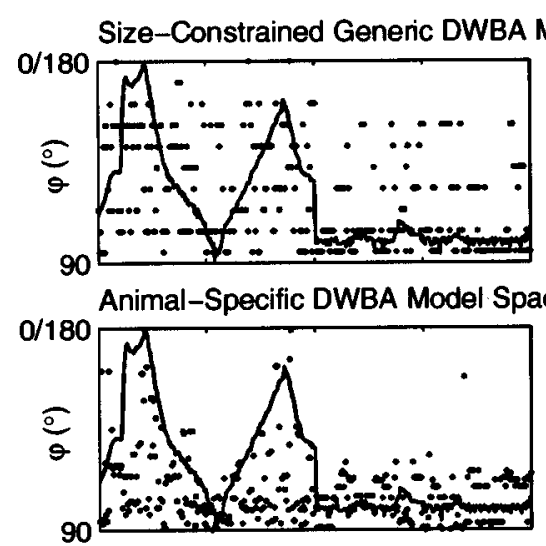

\section{ace}

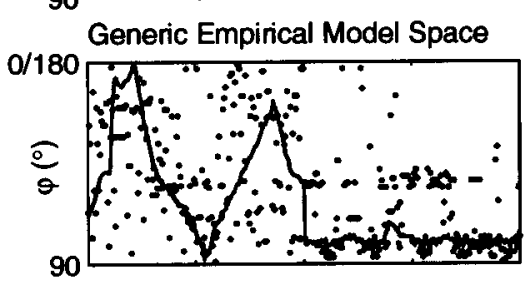

Animal-Specific Empirical Model Space

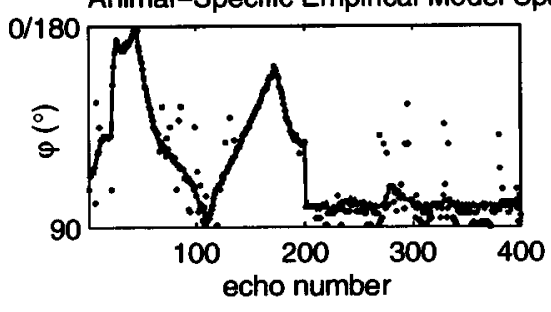

RAW
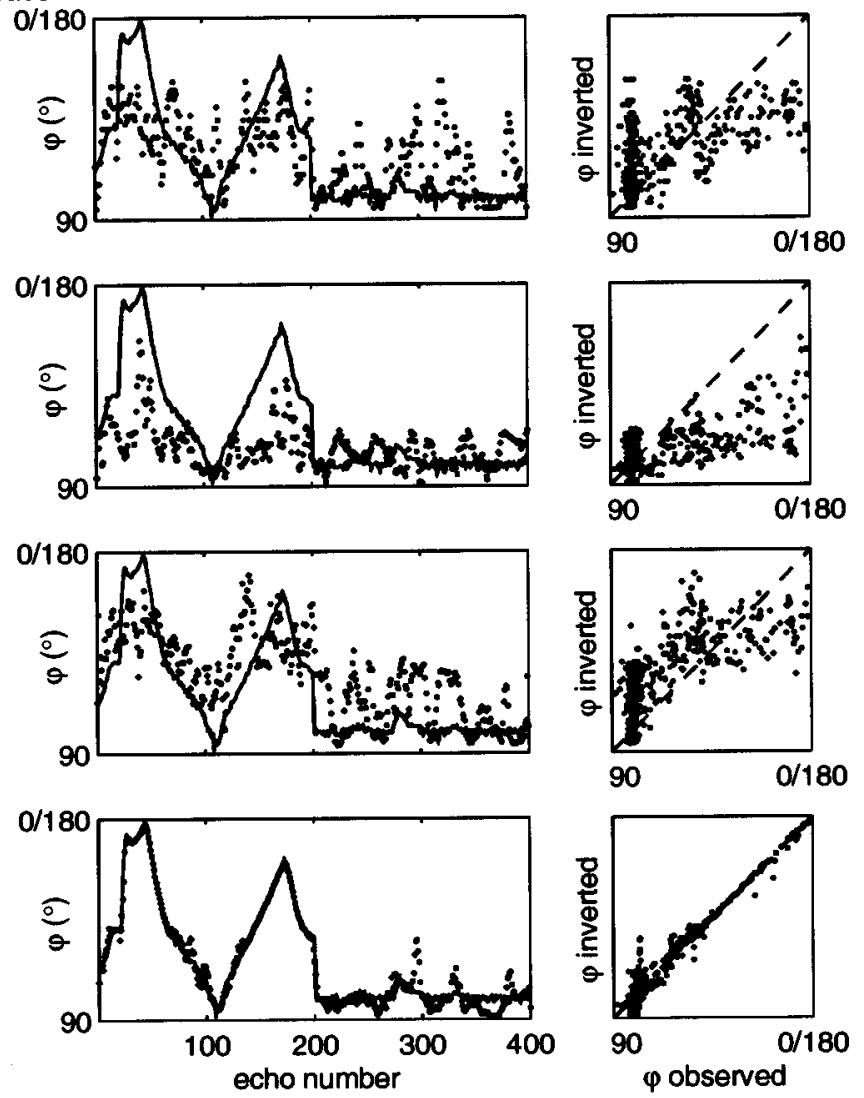

BIN-AVERAGED

FIG. 6. Inversion of echo spectra from Animal 03 for angle of orientation using the CMVC inversion technique, assuming symmetry about broadside incidence $\left(90^{\circ}\right)$. Observed angle of orientation $\varphi$ (solid line) shown together with inversion results (points) achieved using four different model spaces: generic DWBA model space constrained to approximate size of Animal 03 (top); animal-specific DWBA model space for Animal 03 (2nd row); generic empirical model space (based on data from Animal 01, 3rd row); animal-specific empirical model space (based on data from Animal 03, bottom). Raw results shown at left, bin-averaged (over five echoes) results shown at right, including scatter plot of inverted versus observed angle of orientation (dashed $45^{\circ}$ line indicates perfect correspondence between inversion results and observations).

and the theoretical model predictions for all animals exhibited considerable symmetry around $90^{\circ}$ [see Figs. 4 and 5, parts $(\mathrm{F})$ and $(\mathrm{G})]$, so that a good match to a $45^{\circ}$ model realization will likely also be a good match to the similar $135^{\circ}$ model realization. In applying the CMVC inversion algorithm, the mean similarity [as represented by $\mathbf{X}$ in Eq. (5)] was included only for inversions using the animal-specific empirical model space. This mean comparison was suppressed when classifying with the other three model spaces, since the theoretical models had been shown to under-predict mean echo levels at many angles of orientation, and an em- pirical model based on a different-sized animal will exhibit different mean echo levels. In these cases, the inversion was based only on correlation in spectral structure between the observed echo spectra and the model realizations in the model space, as well as the variance similarity of the observations and the model realizations.

For elongated, fluid-like zooplankton such as krill, the structure of the frequency response depends on both size and orientation, so that it is not possible to invert broadband echo spectra for angle of orientation without some a priori information regarding animal size. In fact, animal size and angle

TABLE II. Performance (percent correct, to within measurement uncertainty of $\pm 15^{\circ}$ ) based on bin-averaged (over five echoes) inversion results (assuming symmetry about broadside incidence) for all 11 krill with the generic DWBA model space (constrained to approximate size of animal), the appropriate animal-specific DWBA model space, the generic empirical model space (based on data from Animal 01) and the appropriate animal-specific empirical model space (Animal 01, 03, 05, and 09 only).

\begin{tabular}{|c|c|c|c|c|c|c|c|c|c|c|c|}
\hline \multirow[b]{2}{*}{ Model space } & \multicolumn{11}{|c|}{ Animal number } \\
\hline & 01 & 02 & 03 & 04 & 05 & 06 & 07 & 08 & 09 & 10 & 11 \\
\hline Generic DWBA & $40 \%$ & $61 \%$ & $63 \%$ & $78 \%$ & $48 \%$ & $62 \%$ & $70 \%$ & $60 \%$ & $35 \%$ & $60 \%$ & $36 \%$ \\
\hline Animal-specific DWBA & $30 \%$ & $52 \%$ & $69 \%$ & $33 \%$ & $35 \%$ & $48 \%$ & $57 \%$ & $54 \%$ & $34 \%$ & $43 \%$ & $34 \%$ \\
\hline Generic empirical & $78 \%$ & $65 \%$ & $57 \%$ & $74 \%$ & $61 \%$ & $70 \%$ & $38 \%$ & $47 \%$ & $51 \%$ & $66 \%$ & $66 \%$ \\
\hline Animal-specific empirical & $78 \%$ & $\cdots$ & $99 \%$ & $\cdots$ & $90 \%$ & $\cdots$ & $\cdots$ & $\cdots$ & $97 \%$ & $\cdots$ & $\cdots$ \\
\hline
\end{tabular}



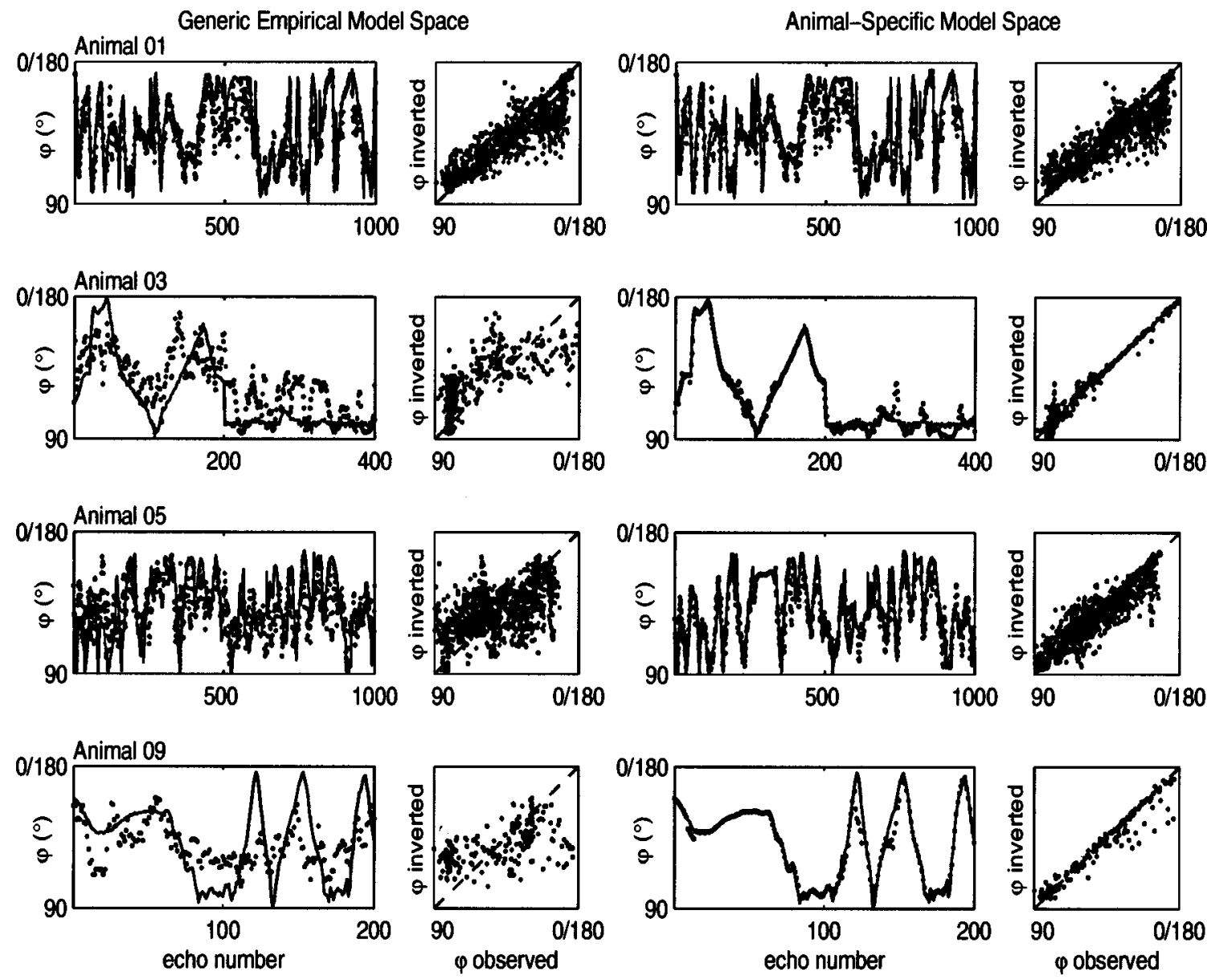

FIG. 7. Bin-averaged (over five echoes) inversion results for Animal 01, 03, 05, and 09 (top to bottom) using the CMVC inversion technique (assuming symmetry about $90^{\circ}$ ) with the generic empirical model space (based on data from Animal 01, left) and the animal-specific empirical model space (right). Observed orientation $\varphi$ (solid line) shown together with inversion results (points); $45^{\circ}$ dashed line in scatter plot indicates perfect agreement between inversion results and observations.

of orientation are confounded, so that the frequency response of an echo received from a large animal may have a structure similar to that received from a much smaller animal at a different orientation relative to the incident acoustic wave. To illustrate this, consider a simple scattering model which includes a summation of only two rays (Stanton et al., 1993a,b), accounting for the constructive and destructive interference between the rays reflected from the front and back interfaces of a weakly scattering target such as a krill. The null spacing of the frequency response predicted by this tworay model depends on the apparent size of the animal, that is, the distance the acoustic wave travels between the front and back interfaces of the animal. Apparent size is a function of both animal radius and angle of orientation, so that the echo spectrum of a large krill at broadside incidence can exhibit the same structure as that of a smaller animal oriented off-broadside relative to the direction of insonification (Fig. 8).

Knowledge of animal orientation during insonification could significantly improve acoustic biomass estimates of zooplankton, particularly for aggregations of similarly sized individuals of a single species, for example, swarms of Antarctic krill in the Southern Ocean. The in situ orientation distribution of Euphausia superba has not been measured quantitatively. Observations of freely swimming E. superba in an aquarium indicated that they assumed a wide range of orientations (corresponding to $\varphi$ varying between $40^{\circ}$ and $180^{\circ}$ ), but spent most of the time swimming upward at a steep angle, so that they would most often be oriented within $60^{\circ}$ of end-on incidence relative to a downward-looking echosounder (Kils, 1981). It is probable that the animals in Kils' aquarium (as well as the tethered krill insonified in these scattering experiments) assumed a much wider range of orientations than would be observed in the field by a downward-looking sonar system. In fact, qualitative in situ observations of $E$. superba by Hamner et al. (1983) revealed that all individuals in a school assumed the same orientation, and that krill in an aggregation usually swam horizontally; descending at angles of less than approximately $10^{\circ}$ relative to the horizontal.

Application of the CMVC inversion technique in the field could potentially allow prediction of the orientation of individual elongated, fluid-like zooplankton (e.g., krill) in situ. Information from broadband acoustic systems, combined with ground-truthing of animal size (e.g., from net samples), could be inverted for angle of orientation with the CMVC inversion technique using a generic empirical model space (e.g., the one constructed based on data collected from Animal 01, or alternatively, one based on data collected from krill at known orientations in situ). Certain technological 
CASE 1:

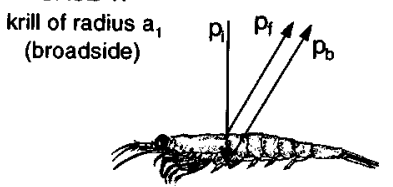

CASE 2:

krill of radius a (broadside)
CASE 3:

krill of radius a (off-broadside)

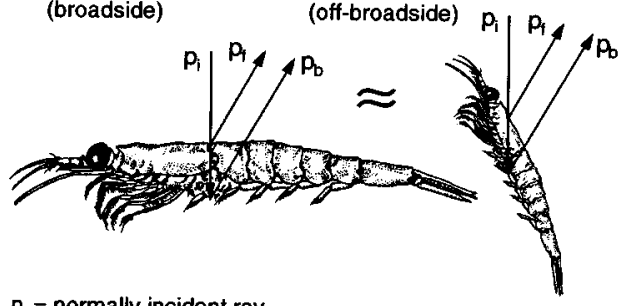

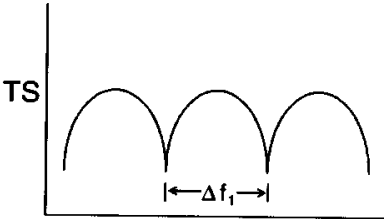

frequency

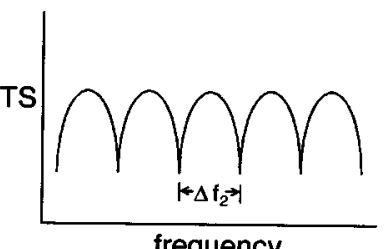

$p_{i}=$ normally incident ray

$\mathrm{P}_{\mathrm{f}}=$ ray scattered from front interface (in receiver direction)

$p_{b}=$ ray scattered from back interface (in receiver direction)
FIG. 8. Comparison of echo spectra predicted by a simplified two-ray theoretical scattering model [e.g., Stanton et al. $(1993 \mathrm{a}, \mathrm{b})]$ received from krill insonified at high frequency $(\lambda \leqslant 4 a)$ for a small animal (radius $a_{1}$ ) at broadside incidence (CASE 1) and off-broadside (CASE 3), and a large animal (radius $a_{2}>a_{1}$ ) at broadside incidence (CASE 2). Nulls in the echo spectra result from destructive interference between the echo from the front interface $\mathrm{p}_{\mathrm{f}}$ and the echo from the back interface $\mathrm{p}_{\mathrm{b}}$ of the animal, which occurs when the phase difference between them $\phi_{\mathrm{f} / \mathrm{b}}$ is $n \pi$ radians; for the three cases shown: $\phi_{\mathrm{f} / \mathrm{b}}=n \pi=\left(2 \pi \Delta \mathrm{f}_{1} / c\right) \mathrm{d}_{1}$ $=\left(2 \pi \Delta \mathrm{f}_{2} / c\right) \mathrm{d}_{2}=\left(2 \pi \Delta \mathrm{f}_{3} / c\right) \mathrm{d}_{3}$, where $\mathrm{d}$ is the distance the ray travels inside the body in each case, and $c$ is the speed of sound in the body. When an animal is at broadside incidence (CASE 1 and CASE 2), $\mathrm{d}_{1}=4 \mathrm{a}_{1}$ and $\mathrm{d}_{2}=4 \mathrm{a}_{2}$, but off-broadside (CASE 3), the apparent size (to the acoustic wave) of the small krill is greater than $\mathrm{a}_{1}$; i.e., $\mathrm{d}_{3} \neq \mathrm{d}_{1}$. Here $\mathrm{d}_{3} \approx \mathrm{d}_{2}$ so that the null spacing is the same for CASE 3 as for CASE 2 (i.e., $\Delta \mathrm{f}_{3} \approx \Delta \mathrm{f}_{2}$ ). challenges must be overcome to permit successful field implementation of a classification inversion for animal orientation based on broadband echo spectra. These include variable beam width and variable signal-to-noise ratio (SNR) over the bandwidth of current broadband sources suitable for field use. Development of constant beam width broadband transducers is underway by others. Orientational information obtained through inversion of the frequency response of broadband echoes may then be used in conjunction with single-frequency acoustic survey data to make more accurate biomass estimates of Antarctic krill stocks in the Southern Ocean.

\section{SUMMARY}

Biomass estimates of Antarctic krill (Euphausia superba) stocks in the Southern Ocean are often based on acoustic survey data. To make accurate estimates of zooplankton biomass from acoustic backscatter measurements, the acoustic characteristics of the species of interest must be well-understood. In particular, it has long been recognized that the acoustic target strength (TS) of elongated, fluid-like zooplankton such as E. superba varies with animal orientation. Acoustic scattering experiments were performed to elucidate the effect of animal orientation on the broadband scattering characteristics of Antarctic krill. During the experiments, individual, live krill were tethered and suspended in a tank filled with filtered, chilled seawater. One thousand echoes were collected from each of 14 animals during insonification with a broadband chirp of center frequency $500 \mathrm{kHz}(\sim 350-750 \mathrm{kHz})$, while their behavior was simultaneously captured on video tape. A novel video analysis technique was applied to images for 11 of the animals to extract the angle of orientation of the krill corresponding to each insonification. This analysis revealed that echo spectra from krill near broadside incidence relative to the incident acoustic wave were characterized by widely spaced $(\sim 200$ $\mathrm{kHz})$ deep $(\sim 20 \mathrm{~dB})$ nulls, whereas off-broadside echo spectra exhibited a more erratic structure, with several closely spaced $(<50 \mathrm{kHz})$ nulls of variable depth. Spectrally averaged echo levels were found to be about $5 \mathrm{~dB}$ higher near broadside incidence compared to off-broadside. The acoustic returns collected from the krill were compared to theoretical predictions for all angles of orientation based on a distorted wave Born approximation (DWBA) model for each animal. The pattern of changes in echo spectra with orientation is very similar for the experimentally measured data and the DWBA model predicted spectra for all 11 krill. However, the theoretical model predicts a much greater (by about $15 \mathrm{~dB}$ ) drop in echo levels as orientation changes from broadside incidence to off-broadside than was actually observed for these animals as they changed orientation. Information contained in the broadband echo spectra of the krill was exploited to invert the acoustic returns for angle of orientation by applying a newly developed Covariance Mean Variance Classification (CMVC) approach, using generic and animalspecific theoretical and empirical model spaces. The animalspecific empirical model space (based on data collected from the appropriate animal) was best able to invert for angle of orientation. Employing a generic empirical model space (based on data collected from an arbitrarily chosen krill) resulted in more accurate inversions overall than could be achieved using the appropriate animal-specific theoretical model space (which predicts the scattering based on the actual size and shape of that particular animal). The CMVC inversion technique can be implemented using a generic empirical model space to determine angle of orientation based on broadband echoes from individual zooplankton in the field. Pending technological development of a broadband sonar for deployment in conjunction with single-frequency acoustic surveys of Antarctic krill, extraction of this orientational information has the potential to significantly improve biomass estimates of krill stocks in the Southern Ocean.

\section{ACKNOWLEDGMENTS}

The authors would like to thank Chuck Greene for coordinating the 1995 and 1996 Bioacoustical Oceanography Workshops, funded by the National Science Foundation and the Office of Naval Research, during which the scattering experiments and some of the data analysis were conducted. Many people contributed significantly to this work during 
the workshops, especially Karen Fisher, Janice Jones, Andrew Remsen, and Kathy Vigness. Thanks also to Langdon Quetin and Robin Ross of UCSB for providing the krill, Dan Costa, Bernie LeBoeuf, and Betsy Steele for providing laboratory space at UCSC, Bill Lange at the Woods Hole Oceanographic Institution for providing the video equipment, and three anonymous reviewers for helpful suggestions for improvement of the manuscript. This work was completed in partial fulfillment of the requirements for a Ph.D., and L.V.MT. would like to thank her thesis committee: Tim Stanton (who also provided the pulse-echo acoustic data acquisition system and guidance regarding the theoretical models), Peter Wiebe and Jim Lynch at WHOI, and Penny Chisholm of the Massachusetts Institute of Technology, for their support, encouragement, and advice over the last five years. L.V.MT.'s research was supported by the Ocean Acoustics, Oceanic Biology and URIP programs of the Office of Naval Research Grant Nos. N00014-89-J-1729, N00014-95-1-0287, and N00014-92-J-1527, the Biological Oceanography program of the National Science Foundation Grant No. OCE-9201264, and the WHOI/MIT Joint Program Education Office. R.L.O. would like to thank Television New Zealand and the Claude McCarthy Fellowship Foundation for supporting his participation in the workshops. D.E.M. would like to thank Tracor and the WHOI Postdoctoral Fellowship program for support. This is Woods Hole Oceanographic Institution contribution number 9596.

Anderson, V. C. (1950). "Sound scattering from a fluid sphere,' J. Acoust. Soc. Am. 22, 426-431.

Chu, D., Stanton, T. K., and Wiebe, P. H. (1992). "Frequency dependence of sound backscattering from live individual zooplankton,' ICES J. Mar. Sci. 49, 97-106.

Chu, D., Foote, K. G., and Stanton, T. K. (1993). "Further analysis of target strength measurements of Antarctic krill at 38 and $120 \mathrm{kHz}$ : Comparison with deformed cylinder model and inference of orientation distribution,', J. Acoust. Soc. Am. 93, 2855-2988.

Clay, C. S., and Medwin, H. (1977). Acoustical Oceanography: Principles and Applications (Wiley, New York).

Croxall, J. P., McCann, T. S., Prince, P. A., and Rothery, P. (1988). "Reproductive performance of seabirds and seals at South Georgia and Signy Island, South Orkney Islands, 1976-1987: Implications for Southern Ocean monitoring studies,', in Antarctic Ocean and Resources Variability, edited by D. Sahrhage (Springer-Verlag, New York).

Demer, D. A., and Martin, L. V. (1995). "Zooplankton target strength: Volumetric or areal dependence?,' J. Acoust. Soc. Am. 98, 1111-1118. El-Sayed, S. Z. (1988). “'The BIOMASS program,', Oceanus 31, 75-79.

Everson, I. (1977). The living resources of the Southern Ocean. Food and Agriculture Organisation of the United Nations, GLO/SO/77/1, Rome, 156 pp.

Everson, I. (1982). "Diurnal variations in mean volume backscattering strength of an Antarctic krill (Euphausia superba) patch,' J. Plankton Res. 4, 155-162.

Everson, I., Watkins, J. L., Bone, D. G., and Foote, K. G. (1990). "'Implications of a new acoustic target strength for abundance estimates of Antarctic krill,' Nature (London) 345, 338-340.

Flagg, C. N., and Smith, S. L. (1989). "'On the use of the acoustic Doppler current profiler to measure zooplankton abundance,', Deep-Sea Res. Oceanogr. Abstr. 36, 455-474.

Foote, K. G. (1990). 'Speed of sound in Euphausia superba,' J. Acoust. Soc. Am. 87, 1405-1408.

Foote, K. G., Everson, I., Watkins, J. L., and Bone, D. G. (1990). “'Target strengths of Antarctic krill (Euphausia superba) at $38 \mathrm{kHz}$ and $120 \mathrm{kHz}$,', J. Acoust. Soc. Am. 87, 16-24.

Greene, C. H., Stanton, T. K., Wiebe, P. H., and McClatchie, S. (1991). "Acoustic estimates of Antarctic krill,', Nature (London) 349, 110.
Greenlaw, C. F. (1977). "'Backscattering spectra of preserved zooplankton,', J. Acoust. Soc. Am. 62, 44-52.

Greenlaw, C. F. (1979). "Acoustical estimation of zooplankton populations," Limnol. Oceanogr. 24, 226-242.

Hamner, W. M., Hamner, P. P., Strand, S. W., and Gilmer, R. W. (1983). "Behaviour of Antarctic krill, Euphausia superba: chemoreception, feeding, schooling and molting,', Science 220, 433-435.

Hewitt, R. P., and Demer, D. A. (1991). 'Krill abundance,' Nature (London) 353, 310 .

Hewitt, R. P., and Demer, D. A. (1996). "Lateral target strength of Antarctic krill,', ICES J. Marine Sci. 53, 297-302.

Huntley, M. E., and Lopez, M. D. G. (1992). "Temperature dependent production of marine copepods: a global synthesis,', Am. Nat. 140, 201242.

Johnson, R. K. (1977). "Sound scattering from a fluid sphere revisited,' J. Acoust. Soc. Am. 61, 375-377.

Kils, U. (1981). "The swimming behaviour, swimming performance and energy balance of Antarctic krill, Euphausia superba,' BIOMASS Scientific Series 3.

Kristensen, A., and Dalen, J. (1986). "Acoustic estimation of size distribution and abundance of zooplankton,', J. Acoust. Soc. Am. 80, 601-611.

Martin, L. V., Stanton, T. K., Wiebe, P. H., and Lynch, J. F. (1996). “Acoustic classification of zooplankton,', ICES J. Marine Sci. 53, 217224.

Martin Traykovski, L. V., Lynch, J. F., Stanton, T. K., and Wiebe, P. H. (1998). "Model-based Covariance Mean Variance Classification techniques: Algorithm development and application to the acoustic classification of zooplankton,', IEEE J. Oceanic Eng. 23 (4).

McGehee, D. E., O’Driscoll, R. L., and Martin Traykovski, L. V. (in press). "Effects of orientation on acoustic scattering from Antarctic Krill,', DeepSea Res. Oceanogr. Abstr.

Miller, C. B., and Judkins, D. C. (1981). “Design of pumping systems for sampling zooplankton, with descriptions of two high-capacity samplers for coastal studies," Biol. Ocean. 1, 29-56.

Morse, P. M., and Ingard, K. U. (1968). Theoretical Acoustics (Princeton U.P., Princeton, NJ).

Nemoto, T., Okiyama, M., Iwasaki, N., and Kikuchi, T. (1988). "Squid as predators on krill (Euphausia superba) and prey for sperm whales in the Southern Ocean,' in Antarctic Ocean and Resources Variability, edited by D. Sahrhage (Springer-Verlag, New York), pp. 292-296.

Nicol, S., and de la Mare, W. (1993). "Ecosystem management and the Antarctic Krill,', Am. Sci. 81, 36-47.

Papoulis, A. (1991). Probability, Random Variables, and Stochastic Processes (McGraw-Hill, New York), 3rd ed.

Penrose, J. D., and Kaye, G. T. (1979). " Acoustic target strengths of marine organisms,', J. Acoust. Soc. Am. 65, 374-380.

Permitin, Y. E. (1970). "The consumption of krill by Antarctic fishes," in Antarctic Ecology Vol. I, edited by M. W. Holdgate (Academic, New York), pp. 177-182.

Sameoto, D. D. (1980). "Quantitative measurements of euphausiids using a $120 \mathrm{kHz}$ sounder in their in situ orientation,' Can. J. Fisheries Aquatic Sci. 37, 693-702.

Samovol'kin, V. G. (1980). "Backscattering of ultrasonic waves by shrimps,", Oceanology 20, 667-670.

Stanton, T. K. (1988a). "Sound scattering by cylinders of finite length. I. Fluid cylinders,', J. Acoust. Soc. Am. 83, 55-63.

Stanton, T. K. (1988b). "Sound scattering by cylinders of finite length. II. Elastic cylinders,', J. Acoust. Soc. Am. 83, 64-67.

Stanton, T. K. (1989a). "Sound scattering by clinders of finite length. III. Deformed cylinders,', J. Acoust. Soc. Am. 86, 671-705.

Stanton, T. K. (1989b). "Simple approximate formulas for backscattering of sound by spherical and elongated objects,', J. Acoust. Soc. Am. 86, 14991510.

Stanton, T. K. (1990). "Sound scattering by spherical and elongated shelled bodies,' J. Acoust. Soc. Am. 88, 1619-1633.

Stanton, T. K., Clay, C. S., and Chu, D. (1993a). "Ray representation of sound scattering by weakly scattering deformed fluid cylinders: Simple physics and application to zooplankton,' J. Acoust. Soc. Am. 94, 34543462 .

Stanton, T. K., Chu, D., Wiebe, P. H., and Clay, C. S. (1993b). "Average echoes from randomly oriented random-length finite cylinders: Zooplankton models,', J. Acoust. Soc. Am. 94, 3463-3472.

Stanton, T. K., Wiebe, P. H., Chu, D., Benfield, M. C., Scanlon, L., Martin, 
L. V., and Eastwood, R. L. (1994a). "On acoustic estimates of biomass," ICES J. Mar. Sci. 51, 505-512.

Stanton, T. K., Wiebe, P. H., Chu, D., and Goodman, L. (1994b). “Acoustic characterization and discrimination of marine zooplankton and turbulence,'” ICES J. Mar. Sci. 51, 469-479.

Stanton, T. K., Chu, D., and Wiebe, P. H. (1996). "Acoustic scattering characteristics of several zooplankton groups," ICES J. Mar. Sci. 53, 289-295.

Stanton, T. K., Chu, D., Wiebe, P. H., Martin, L. V., and Eastwood, R. L. (1998a). "Sound scattering by several zooplankton groups I: Experimental determination of dominant scattering mechanisms," J. Acoust. Soc. Am. 103, 225-235.

Stanton, T. K., Chu, D., and Wiebe, P. H. (1998b). "Sound scattering by several zooplankton groups II: Scattering models," J. Acoust. Soc. Am. 103, 236-253.

Wiebe, P. H., Boyd, S., and Cox, J. L. (1975). "Relationships between zooplankton displacement volume, wet weight, dry weight and carbon," Fishery Bull. 73, 777-786.

Wiebe, P. H., Morton, A. W., Bradley, A. M., Backus, R. H., Craddock, J. E., Cowles, T. J., Barber, V. A., and Flierl, G. R. (1985). "New developments in the MOCNESS, an apparatus for sampling zooplankton and micronekton," Mar. Biol. 87, 313-323.

Wiebe, P. H., Greene, C. H., Stanton, T. K., and Burczynski, J. (1990). "Sound scattering by live zooplankton and micronekton: Empirical studies with a dual-beam acoustical system,' J. Acoust. Soc. Am. 88, 23462360 . 